\title{
Chapter 19 \\ Using Art to Bridge Research and Policy: An Initiative of the United States National Academy of Medicine
}

\author{
Charlee Alexander, Kyra Cappelucci, and Laura DeStefano
}

\subsection{Introduction}

The National Academy of Medicine (NAM) (formerly the Institute of Medicine) was established in 1970. Its mission is to improve health for all by advancing science; accelerating health equity; and providing independent, authoritative, and trusted advice nationally and globally. The NAM is one of three academies that make up the National Academies of Sciences, Engineering, and Medicine (the National Academies) in the United States. Together, the National Academies are private, non-profit institutions that work outside of government to provide objective advice on matters of science, technology, and health. To achieve its mission, the NAM engages in a number of activities, including assembling volunteer study committees comprising some of the world's foremost scientists, scholars, policy makers, and health professionals to address some of society's toughest challenges. The NAM also hosts numerous conferences, workshops, symposia, forums, roundtables, and other gatherings that attract the finest minds in academia and the public and private sectors. The NAM's vision is a healthier future for everyone and prides itself on being:

- An independent, evidence-based scientific advisor.

- A national academy with global scope.

- Committed to catalyzing action and achieving impact.

- Collaborative and interdisciplinary.

- An honorific society for exceptional leaders.

Over the past 2 years, the NAM has piloted a new strategy to engage with stakeholders at the individual and community levels through three nationwide calls for

C. Alexander $(\bowtie) \cdot$ K. Cappelucci $\cdot$ L. DeStefano

National Academy of Medicine, Washington, DC, USA

e-mail: CAlexander@trinitywallstreet.org; Kyra.Cappelucci@ama-assn.org;

ldestefano@nas.edu 
art - two related to the concept of health equity, and one related to clinician wellbeing. The arts offer an essential medium through which people can shape their identities, express their experiences, learn more effectively, and view society more wholly. Art stretches the imagination and connects people to one another and to the world around them. Research shows that the arts can enhance teaching and learning in school settings (Ernest and Nemirovsky 2015; Gullat 2008) and can improve learning outcomes and readiness for employment (National Academies of Sciences, Engineering, and Medicine 2017). The arts can also help people communicate complex ideas more effectively (Pollack and Korol 2013) and can offer an outlet for authentic community engagement. Drawing on these insights, the NAM designed a participatory art approach as a way to foster meaningful communication around the concepts of health equity and clinician well-being, promote inclusivity, elevate underrepresented voices, and more fully understand the challenges and lived experiences of people in diverse communities.

These calls for art were a departure from the NAM's more traditional methods of connecting with key stakeholders. Results from this approach included the collection of invaluable insights from communities, young people, and clinicians around the nation (which the NAM has used to inform the development of its programs), as well as the establishment of productive new connections between the NAM and other organizations. Elements of the approach have also been replicated by other organizations, and the calls for art have served to connect external organizations to one another. Included in this chapter is a description of the NAM's calls for art, the outcomes from those calls, lessons learned, and insights for organizations interested in pursuing a similar path.

\subsection{Visualize Health Equity}

The NAM's first call for art in the fall of 2017-Visualize Health Equity-originated from the NAM Culture of Health Program. This program aims to advance health equity in the United States by furthering the knowledge base, bridging science to action, strengthening community assets, influencing policy reform, and making health a shared value. Health equity is the state in which everyone has the opportunity to attain full health potential and no one is disadvantaged from achieving this potential because of social position or any other socially defined circumstance. Promoting health equity means creating the conditions in which individuals and communities have what they need to enjoy full, healthy lives (National Academies of Sciences, Engineering, and Medicine 2017).

Community voice is an important component of the program, and the goal of this community art project was to encourage more thought and discussion about health equity and the social determinants of health $(\mathrm{SDOH})$ by soliciting and elevating insights directly from people in diverse communities across the United States. $\mathrm{SDOH}$ are conditions in the environments in which people are born, live, learn, work, play, worship, and age that affect a wide range of health, functioning, and 
quality-of-life outcomes and risks (Healthypeople.gov 2019). The hope was that through a creative lens, the NAM could better understand what people across the country see as the most important health challenges and opportunities facing their communities. With this call for art, the NAM aimed to initiate conversations centered on the need for more equitable policies so that everyone has an equal chance to thrive. The NAM also leveraged its position as a trusted national convener and advisor to share insights gleaned from the lived experiences of participating artists with policy makers, academics, and organizational leaders. The images and excerpts below are taken from artwork and statements submitted by artists who contributed to Visualize Health Equity (Figs. 19.1, 19.2, 19.3, and 19.4). Each excerpt provides insight into how artists and communities think about health equity and its importance to their lives.

Listen to Filipino and Mexican farmworkers laugh together over lunch—a right afforded to them under labor laws that, after years of struggle, protect historically excluded agricultural and domestic workers. Observe racially and culturally diverse friends meeting in neighborhoods created by federal housing policies that encourage and support, rather than obstruct and undermine, neighborhood integration. Listen to children of all backgrounds swim together because their community supports each of them and a public pool that welcomes everyone...These are the signs of health. These are the signs of equity. (Connie CagampangHeller, Imagine Belonging, Berkeley, CA)

There is something magical that happens when people can see and hear themselves in their communities. There is a building of agency and sustainable change when people have ownership over the spaces that they occupy and when they see themselves all around them. Creating through a lens of equity will not always be easy. It will take work, require outsideof-the-box thinking and may not always be the most lucrative position, but it is worth it to at least attempt to use our creativity as artists to leave this world a better place for others because we were in it. (Visualize Health Equity submission)

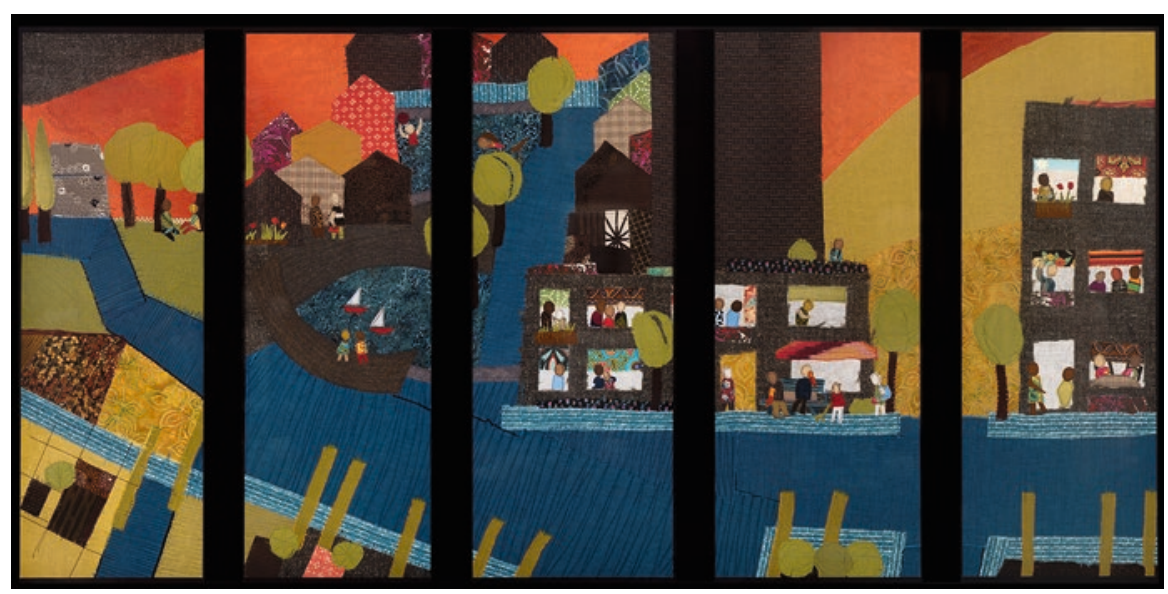

Fig. 19.1 Imagine Belonging, (C) Connie Cagampang Heller, Berkeley, CA; reproduced with permission from the artist from http://nam.edu/visualizehealthequity/\#/. All rights reserved 


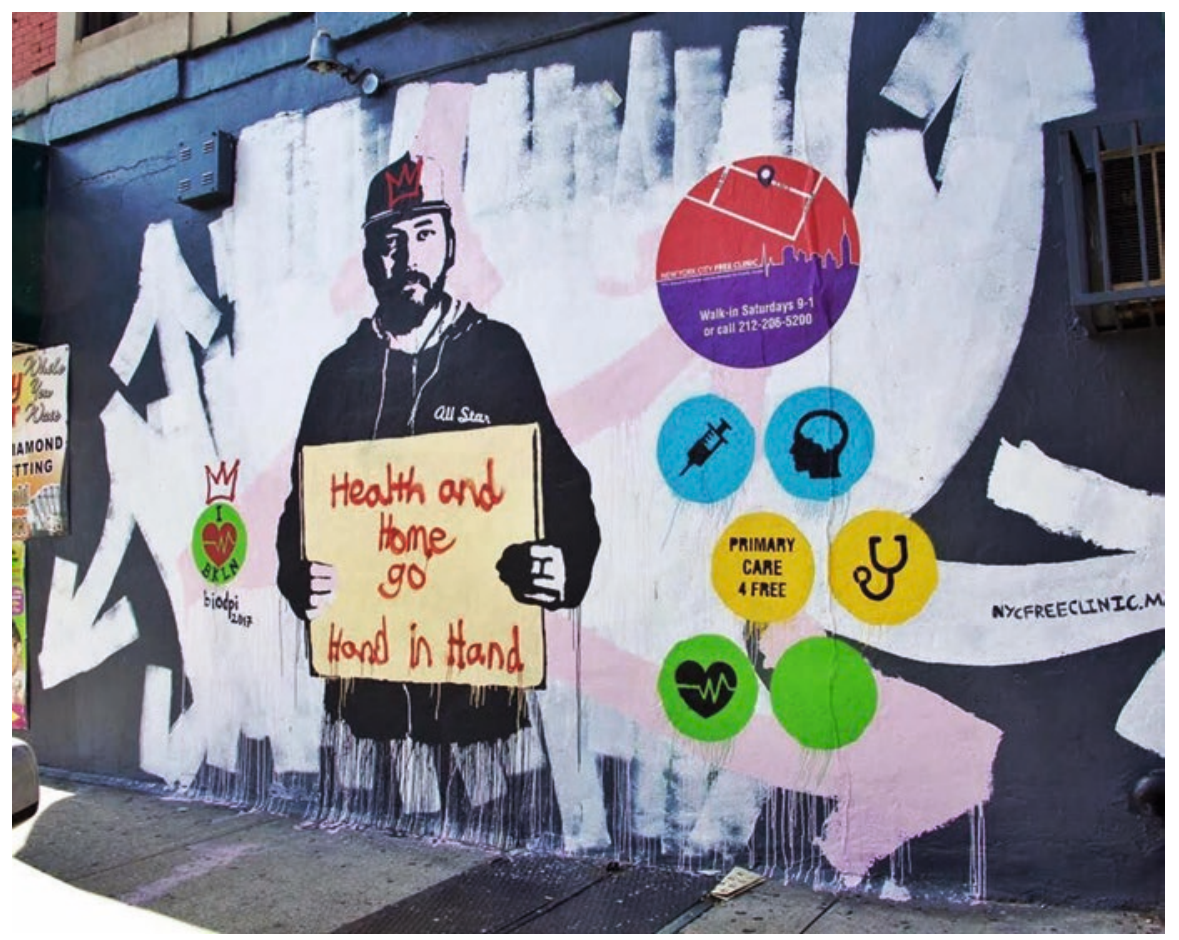

Fig. 19.2 A Picture of Health, (C) John Colavito, New York, NY; Reproduced with permission from the artist from http://nam.edu/visualizehealthequity/\#/. All rights reserved

Visualize Health Equity relied on a simple prompt: "What does health equity look, sound, and feel like to you?" (See Box 19.1 for full text of the call for art.) The NAM distributed the call in June 2017 via listserv announcements, social media, and direct outreach to organizations interested in health equity and communities engaged with the program. Community members from a broad span of geographic locations in the United States were a key audience for this project, as the NAM aimed to shed light on experiences and challenges faced by communities around the nation in achieving health equity. Interested participants had 6 weeks to prepare a submission. Altogether, the NAM received 120 submissions for this inaugural call for art. Submissions came from 29 different states in the United States, Puerto Rico, and Ontario, Canada. People from a broad span of ages, races, genders, socioeconomic status, and abilities submitted pieces highlighting topics including food insecurity, immigration, LGBTQ health and well-being, homelessness, and addiction, among others. Submissions covered a range of media, from drawings and paintings to sculptures and slam poetry. 


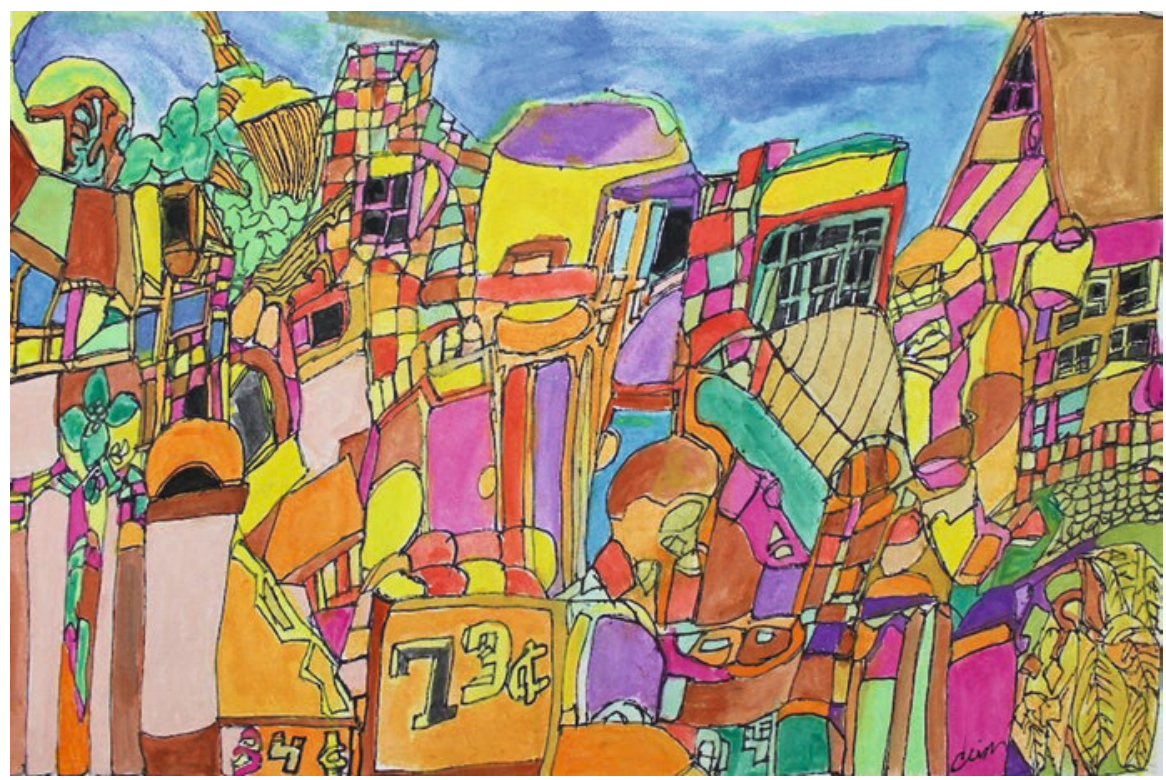

Fig. 19.3 Neighborhood Community, () Egbert "Clem" Evans, Washington, D.C.; Reproduced with permission from the artist from http://nam.edu/visualizehealthequity/\#/. All rights reserved

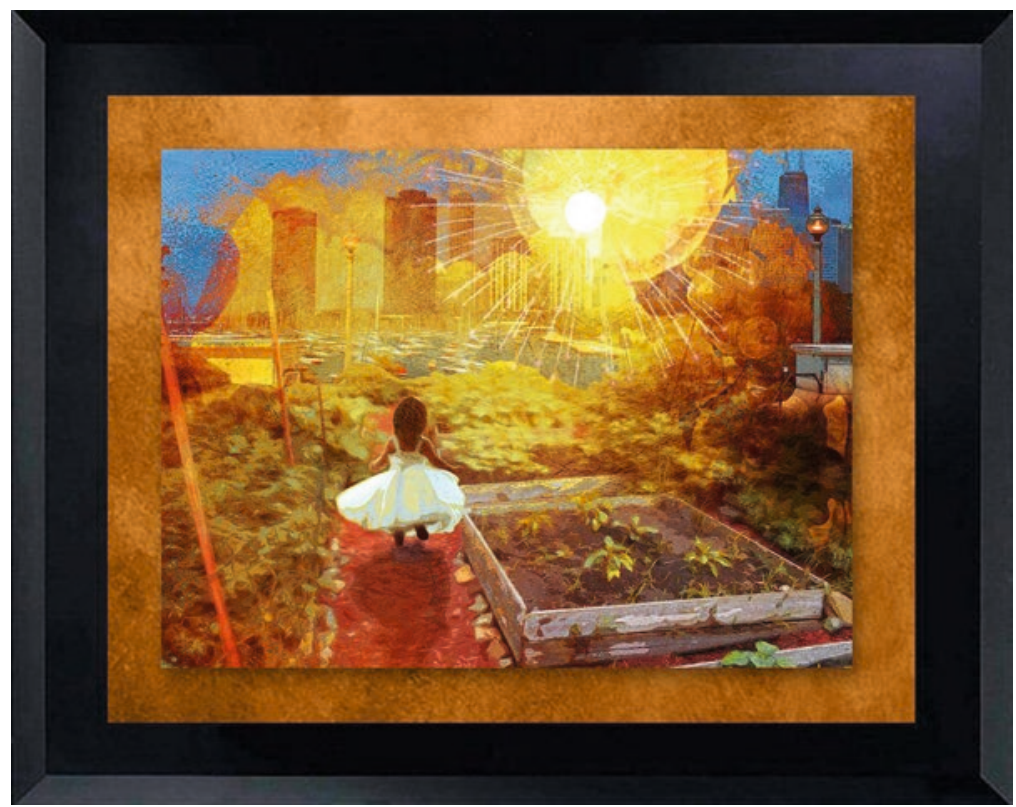

Fig. 19.4 Chasing Sunshine, (C) Stephanie Kohli, Weston, WI; reproduced with permission from the artist from http://nam.edu/visualizehealthequity/\#/. All rights reserved 


\section{Box 19.1 Visualize Health Equity Call for Art}

The National Academy of Medicine (NAM), a non-profit research organization in Washington, DC, is calling on artists to Visualize Health Equity for a nationwide community art project.

Show us what health equity would look like to you-whether it's access to healthy food or safe neighborhoods, good education or a living wage, clean drinking water or affordable housing, connection to cultural heritage or lack of discrimination, or any other opportunity that helps you live your healthiest life. This project is part of the NAM's Culture of Health Program, sponsored by the Robert Wood Johnson Foundation, which is working to identify strategies that support equitable good health for everyone living in the United States. Artwork submitted for this project will help us understand what people across the country see as the most important health challenges and opportunities facing their communities. The insights we gain will be shared with a national audience and used to inform future directions of the Culture of Health Program.

Guidelines:

- Visual and nonvisual art, such as music and creative writing, are welcome. Accepted mediums include drawings, paintings, photographs, mixed media, murals, collage, sculpture, film, poetry, digital art, performance art, and more. Please provide clear photographs of all visual artwork, from multiple angles or showing close-up detail as appropriate. Creative writing should be submitted in PDF format, and performance art or music should be submitted in video format. File size limit: $50 \mathrm{MB}$.

- You may submit previously existing artwork, such as a community art installation, as long as you are the original artist or have formal permission from the artist to submit their work for this project.

- All entries must be accompanied by a completed submission form, which includes a brief written explanation of how your artwork relates to health equity.

- By submitting your artwork for this project, you are granting non-exclusive lifetime permission for the NAM to display, publish, and share your artwork in digital and print formats. This includes the written explanation you submit with your work. Full credit will always be given to the artist, and copyright will remain with the artist. Artwork submitted for this project will not be reproduced for purchase or profit.

- Individuals and groups may participate. Limit three submissions per person/group.

- Artists under the age of 18 must have permission from a parent or guardian to participate.

- Non-English-language submissions are encouraged.

- The NAM reserves the right not to display, publish, or share submissions that are not responsive to the prompt or contain inappropriate language or themes.

SOURCE: National Academy of Medicine (2017) 
A panel of reviewers drawn from the Culture of Health Program advisory committee, a 20-member body providing strategic guidance for the program, evaluated entries for creativity and responsiveness to the prompt. Together, this group reviewed the submissions using the following criteria:

\section{Responsiveness to prompt \\ 2. Uniqueness of insight \\ 3. Visual impact \\ 4. Overall impression}

Reviewers used the overall scores to choose 30 submissions to include as part of an in-person gallery at the NAM and identified four artists to present their work to expert attendees during a NAM convening. All 120 submissions are displayed in a permanent digital gallery accessible from the NAM's website: http://nam.edu/ visualizehealthequity/\#.

Following the launch of the in-person art show and digital gallery, the NAM received numerous inquiries about future calls for art and displaying the submissions at conferences, events, and gallery spaces. In response, NAM developed a free, ten-piece traveling art gallery to promote better understanding of the social determinants of health, illustrate how communities are driving health equity, and encourage communities and organizations to undertake similar initiatives Within the first few weeks of announcing the traveling gallery, the first several months were booked. The traveling gallery has been hosted at six conferences since November 2018, including the South by Southwest (SXSW) Conference and Festival in Austin, Texas, and the annual conference of the American Public Health Association in San Diego, California. Several organizations are scheduled to host the art show throughout 2019. Many others have adopted the NAM's prompt to develop their own calls for art. Information on the traveling exhibit is available at http://nam.edu/visualizehealthequity/\#/traveling-gallery.

Audiences who have viewed the traveling and digital galleries are quick to relate to the experiences represented through the artwork. Many viewers were inquisitive about the term "health equity" and how it relates to their lives. Anecdotal feedback suggests that after viewing the artwork, viewers are able to more clearly explain what health equity means for them and their communities. Viewers find the artwork relatable and can easily see their own lives reflected back to them. Viewing the artwork is often an emotional experience for attendees, with many viewers eager to share insights from their own experiences evoked by the artists' work. At one national conference in late 2018, a viewer commented: "This is my life. This is everything I've been trying to explain to anyone who would listen over the past two decades. I feel validated today."

In early 2019, the NAM launched an additional call for art on the topic of health equity. The new call asked "young leaders" aged 5-26 to imagine a world in which everyone has an equal opportunity to live a healthy life. This call for art aimed to help young people develop a deeper understanding of health equity and to elevate their lived experiences. Please visit the project website for additional details: https:// nam.edu/programs/culture-of-health/young-leaders-visualize-health-equity/. The call for art ended in March 2019 and an online gallery launched in fall 2019. 


\subsection{Expressions of Clinician Well-Being}

Bolstered by the success of Visualize Health Equity, in 2018 the NAM launched a second call for art, this time as part of the Action Collaborative on Clinician WellBeing and Resilience (the Collaborative). The Collaborative aims to improve the well-being of health care professionals by partnering with organizations to raise visibility of clinician anxiety, burnout, depression, stress, and suicide; improve understanding of the factors affecting clinician well-being; and advance evidencebased, multidisciplinary solutions to improve patient care by caring for the caregiver. For this call, the NAM asked stakeholders to submit artwork that expresses what clinician well-being looks, feels, and sounds like to them (see Box 19.2 for full text of the call for art). The ultimate goal was to promote greater awareness and understanding of barriers to the well-being of health professionals, trainees, and students - and of solutions that promise a brighter future.

By raising awareness and elevating the experiences of clinicians, patients, and clinicians' loved ones, the NAM hoped to incite purposeful dialogue on this critical issue and begin to decrease stigma. To engender this dialogue, the call for art was designed to create a supportive environment that promotes social connection and fosters new relationships, as discussed by Urke and colleagues in Chap. 1. There were several key audiences identified for this project. First and foremost, the NAM aimed to hear directly from practicing health professionals about their experiences with burnout and challenges to their overall well-being. Additionally, the NAM sought submissions from patients, because clinician well-being largely affects quality of care and outcomes. By sharing insights directly from clinicians and patients, the NAM strived to extract emotions and stories that would invite an open dialogue about burnout between clinicians and health system leaders and would shed light on an issue of national importance. The images below are taken from artwork submitted by artists who contributed to Expressions of Clinician Well-Being (Figs. 19.5, 19.6, 19.7, and 19.8).

The NAM distributed the call for art via listserv announcements, social media, and direct outreach to clinician well-being stakeholders such as medical schools, residency programs, hospitals and health centers, professional societies, and membership organizations. The NAM also engaged arts-focused organizations from around the country. The NAM received over 350 submissions from clinicians and their loved ones, patients, organizations, and other interested stakeholders. Topics of the artwork spanned the well-being of women physicians, the resilience journey from burnout to well-being, joy in practice, self-preservation, and empathy, among others. Submissions included poetry, paintings, music, videos, creative writing, and more. The excerpts below are taken from several artist statements from Expressions of Clinician Well-Being. Each excerpt accompanies a piece of art and provides insight into how the experiences of these artists help them to conceptualize clinician well-being.

This song speaks to clinician resilience and the journey from burnout to well-being. In the first verse, I compare my experience in Iraq as a medic to the gang violence here on 


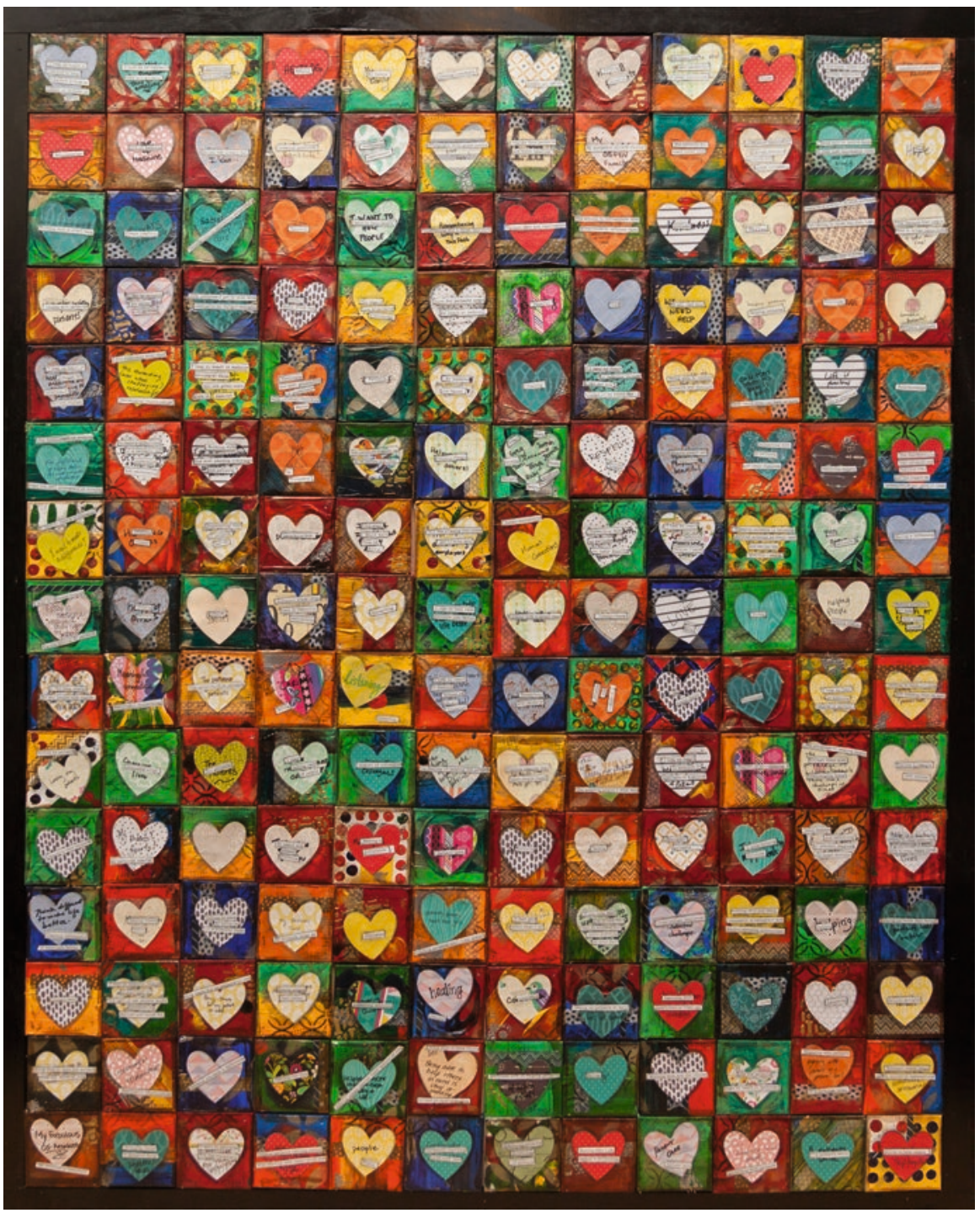

Fig. 19.5 Hearts in Medicine, Residents, fellows, and faculty of the University of Arizona College of Medicine-Phoenix Graduate Medical Education Programs, Phoenix, AZ; reproduced with permission from (C) Cheryl O’Malley from http://nam.edu/expressclinicianwellbeing/\#/. All rights reserved

American soil. In the second verse, a patient addicted to heroin evokes my father's death by a heroin overdose when I was nineteen years old. Entailed in these verses are the emotions that emergency physicians manage every day and the difficulty of debriefing after crises in a busy emergency department. In verse three, I awaken, burned out, and I discuss my transformation from burnout to well-being. (Expressions of Clinician Well-Being submission) 


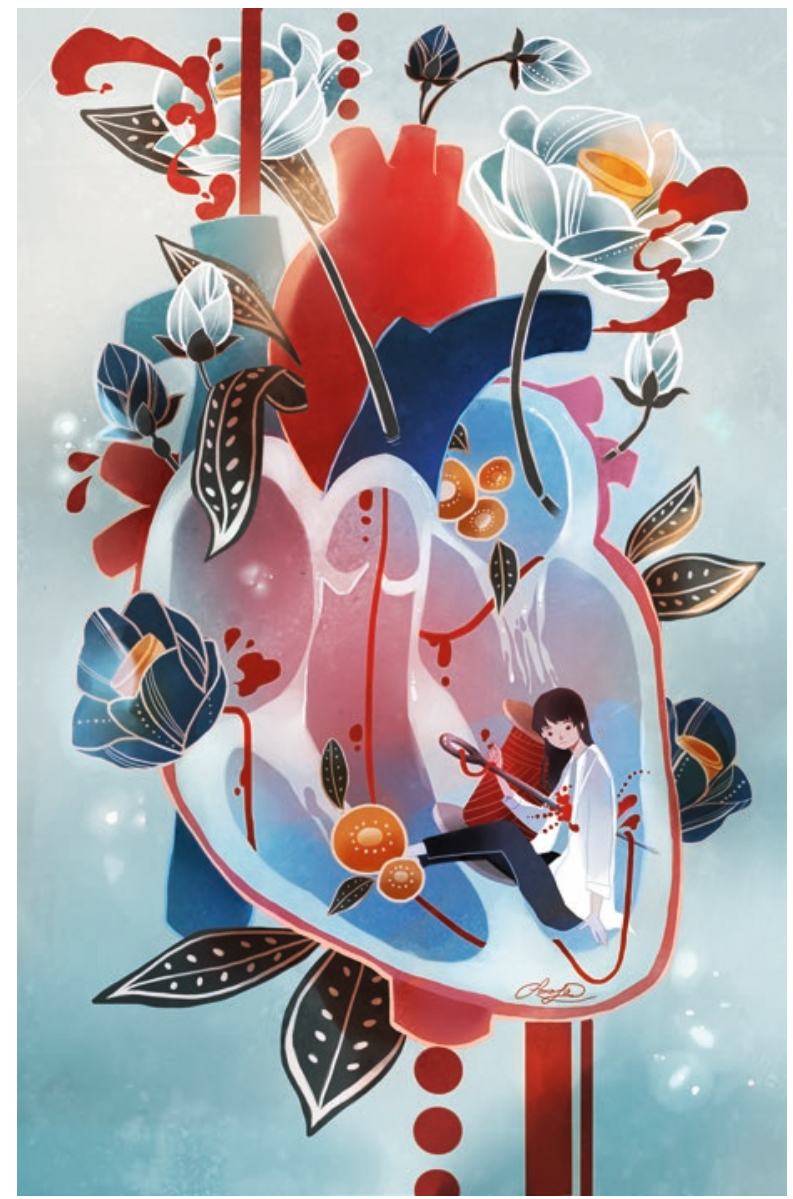

Fig. 19.6 The Red Thread, ( ) Sonia Lai, Fremont, CA; reproduced with permission from the artist from http://nam.edu/expressclinicianwellbeing/\#/. All rights reserved

Dental professionals are mindful of patients' anxiety mostly caused from pain associated with receiving treatment and financial stress related to out-of-pocket costs. The stereotype of dentists being "monsters," rather than heroes, in the health care realm can affect selfworth and self-image. Over time, patient dissatisfaction, pressures of running a successful practice, and paying back high student loans can all lead to clinician burnout, depression, isolation, clinical errors, and decline of overall health. This poem addresses such struggles, but also reminds the reader to self-reflect and draw strength from all the good that comes from the privilege of serving and treating patients. (Expressions of Clinician Well-Being submission)

We choose medicine as a profession in order to help other human beings. It is the connection between practitioner and patient that becomes the foundation of the healing relationship and it is exactly that bond which has inherent in it both the possibility of clinician and patient well-being and the risk of burnout. Too often we think of the doctor-patient relationship as a one-way street. The truth is that we receive as much from our patients as we give to them. (Expressions of Clinician Well-Being submission) 


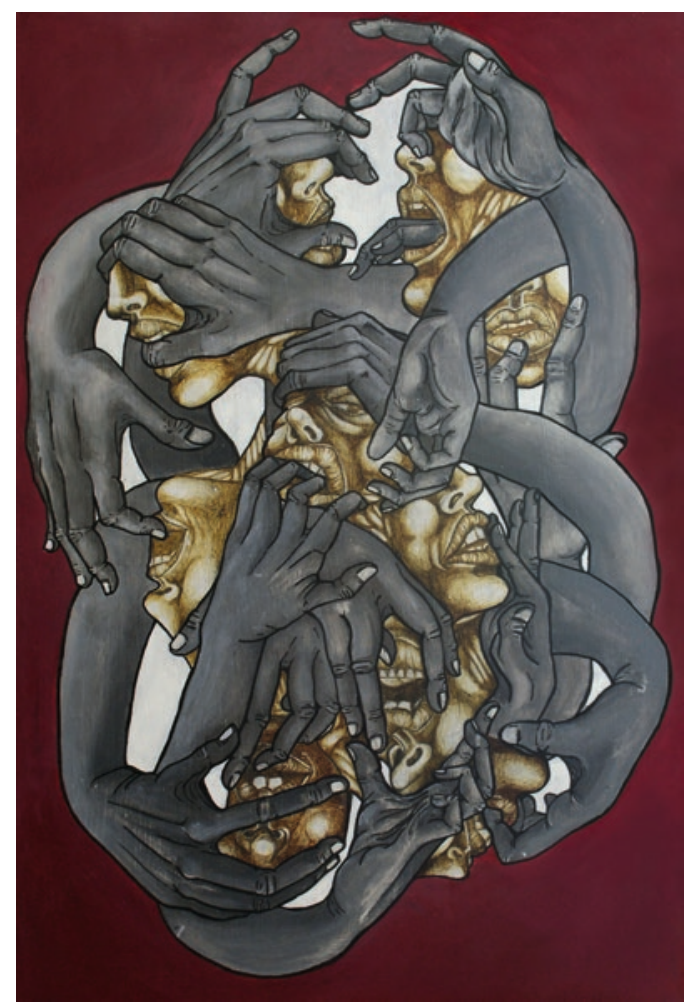

Fig. 19.7 Resilience, (C) Cheyanne Silver, Forest Park, IL; Reproduced with permission from the artist from http://nam.edu/expressclinicianwellbeing/\#/. All rights reserved

\section{Box 19.2 Expressions of Clinician Well-Being Call for Art}

Supporting clinician well-being requires sustained attention and action at organizational, state, and national levels, as well as investment in research and information-sharing to advance evidence-based solutions. More broadly, sustained change requires diverse, collective action and the experiences and voices of many.

Use any art form to show us what clinician burnout, clinician resilience, and/or well-being means to you. Whether it's a depiction of how you de-stress from a busy day, how you feel when taking care of patients, or a picture of your favorite clinician, show us-what does clinician well-being look, feel, and sound like to you? Everyone has a stake in this issue-what's yours?

The well-being of our clinicians impacts everyone. This art show will promote greater awareness and understanding of barriers to clinician wellbeing - and of solutions that promise a brighter future. 


\section{Box 19.2 (continued)}

\section{Participation Guidelines}

[Same as Box 19.1].

\section{Need Inspiration?}

Below are questions to help spark your creativity! These questions are provided as examples only; your submission does not necessarily need to answer one of them.

- If you had the opportunity, how would you express your gratitude to a clinician who provided excellent care to you or a loved one?

- Who has made a difference in your care? How did that clinician make you feel?

- What was your "turning point" in clinician well-being? When did you first start thinking about clinician burnout and well-being? Was there an experience before that turning point that made you think about well-being? How has your life changed since then?

- How do you feel on your worst days versus your best days? What factors affect this?

- How do you support your loved one(s) in taking steps toward achieving a state of well-being?

- How have your loved one's experience(s) with burnout and well-being affected you?

- How does your organization/team/work unit create an atmosphere that supports well-being?

- Why does clinician well-being matter to you? Why should it matter to others?

SOURCE: National Academy of Medicine (2018)

A panel of reviewers drawn from participants of the Action Collaborative on Clinician Well-Being and Resilience evaluated entries for creativity and responsiveness to the prompt, using the same criteria from Visualize Health Equity. Reviewers used the overall scores to choose 30 submissions to include at an in-person gallery at the NAM and identified three artists to present their work to expert attendees during a NAM convening. One hundred of the submissions are displayed in a permanent digital gallery accessible from the NAM's website: http://nam.edu/expressclinicianwellbeing/\#/. This digital collection has been shared broadly with clinicians from around the country through social media and at national medical and health conferences. The Collaborative also asked each of its $190+$ network organizations to share the digital collection with their constituents in an effort to broaden and promote a national dialogue among clinicians and health system leaders on the importance of enacting change to support well-being and prevent burnout. 


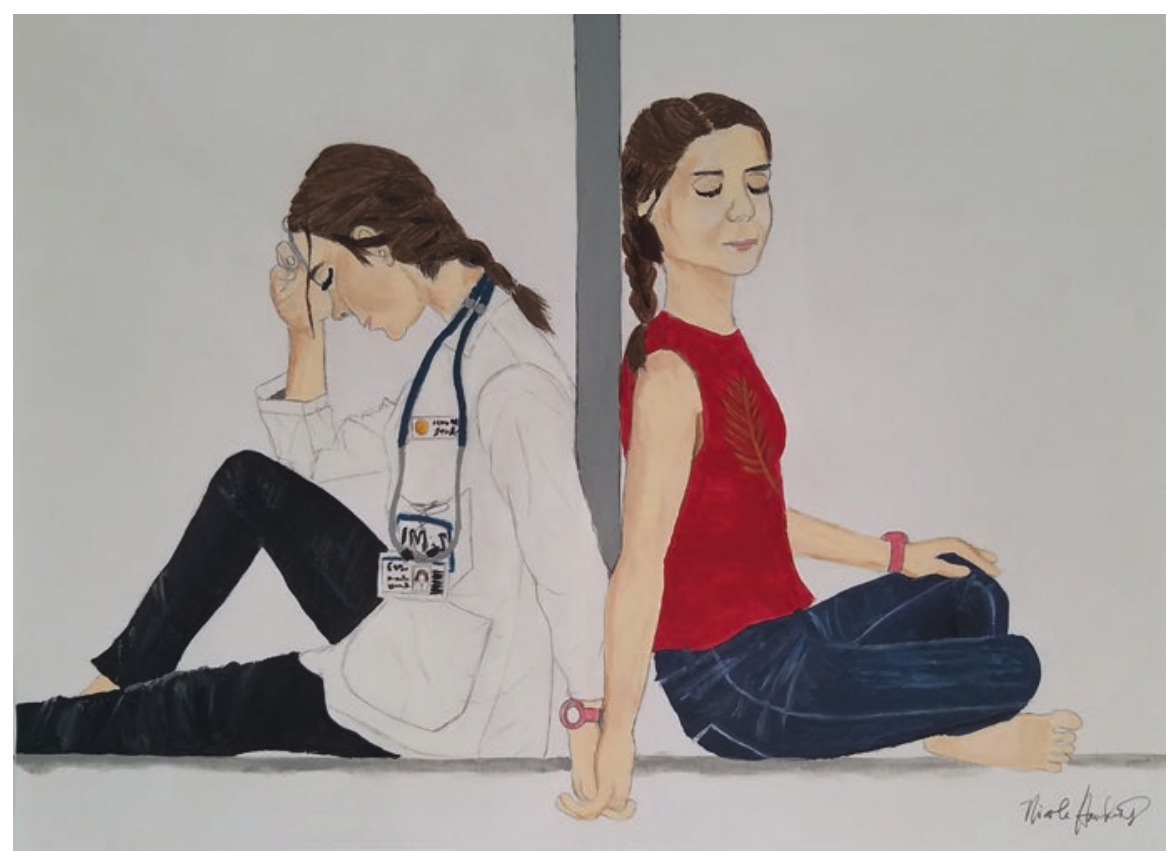

Fig. 19.8 Self-Preservation, (C) Nicole Hawkins, Norfolk, VA; Reproduced with permission from the artist from http://nam.edu/expressclinicianwellbeing/\#/. All rights reserved

Similar to Visualize Health Equity, the NAM created a free, traveling gallery of Expressions of Clinician Well-Being . Viewers of both the traveling and digital galleries are quick to note the validation they feel when viewing the artwork. At one national conference a clinician commented that those who have suffered from burnout for decades may feel as though the artwork gives them "permission to emerge from the shadows" on an issue that has been silenced through stigma for so long. Viewers of the traveling gallery empathize with the artists and their fellow viewers, expressing relief that clinician burnout is finally being discussed openly. Patients who view the artwork often express shock and curiosity; during a NAM convening, a patient noted that he "had no idea clinicians were suffering this way." Expressions of Clinician Well-Being makes clinician burnout and suicide a more visible issue and may help viewers understand and engage with their health care providers on a more personal level.

The gallery has traveled to more than 20 external conferences and events. Additionally, several organizations have hosted their own art show using the NAM's prompt. Artwork from the show has been used for several temporary galleries at universities and hospitals around the country. For additional details, please visit http://nam.edu/expressclinicianwellbeing/\#/traveling-gallery. 


\subsection{Conclusion}

While the NAM is still an institution primarily grounded in advancing science to improve health, these calls for art represent a new way of thinking and engaging with the communities it seeks to serve. In addition to continuing to convene experts and elevate the evidence base, the NAM recognizes the important role that artistic expression can play in fully understanding the lived experiences of people so that policies and programs are responsive to their actual needs, a concept that is further explored by Corbin and colleagues in Chap. 20. The submissions gathered through this strategic and purposeful effort deepen our collective understanding of the lived experiences of those living in the United States and the global community. We heard from an Iraq war veteran-turned-emergency room doctor how his experiences in war are similar to the crises he deals with daily in the emergency room (Rise Up Now, Expressions of Clinician Well-Being). A young student from St. Louis, Missouri, shared her insights on how a single highway effectively ensures the separation of affluence and poverty, deepening disparities and inequities (Delmar Divide No More, Visualize Health Equity). A California artist used a stitched collage to illustrate the importance of racially and culturally diverse communities in strengthening overall well-being and health (Imagine Belonging, Visualize Health Equity). A medical student shared the hardships she has faced because of her gender and how fellow physicians sometimes feel smothered and trapped by the myriad outside influences that inhibit their ability to provide the best patient care (Resilience, Expressions of Clinician Well-Being). Future calls for art might include a follow up mechanism to understand what effects the art shows have on participants in terms of new knowledge, ideas, or behavior.

The snapshots provided here barely scratch the surface of the robust perspectives collected through the art projects, but they provide important insights that are necessary for the academic community, policy makers, and others in understanding the lived realities of so many people. By understanding these realities more fully, stakeholders can more strategically direct their action and resources. Art has offered a way for the NAM to foster new relationships, raise awareness of critical issues, and engage stakeholders in meaningful dialogue around issues that greatly affect the health and well-being of all.

\section{References}

Ernest, J. B., \& Nemirovsky, R. (2015). Arguments for integrating the arts: Artistic engagement in an undergraduate foundations of geometry course. Primus, 26(4), 356-370. https://doi.org/10. 1080/10511970.2015.1123784.

Gullatt, D. E. (2008). Enhancing student learning through arts integration: Implications for the profession. The High School Journal, 91(4), 12-25.

HealthyPeople.gov. (2019). Social determinants of health. https://www.healthypeople.gov/2020/ topics-objectives/topic/social-determinants-of-health. Accessed 25 Feb 2019. 
National Academies of Sciences, Engineering, and Medicine. (2017). Communities in action: pathways to health equity. Washington, DC: The National Academies Press. https://doi. org/10.17226/24624.

National Academy of Medicine. (2017). Visualize health equity: A community art project. http:// nam.edu/visualizehealthequity/\#/. Accessed 25 Feb 252019.

National Academy of Medicine. (2018). Expressions of clinician well-being: an art exhibition. https://nam.edu/expressions-of-clinician-well-being-an-art-exhibition/. Accessed 25 Feb 2019.

Pollack, A. E., \& Korol, D. L. (2013). The use of haiku to convey complex concepts in neuroscience. Journal of Undergraduate Neuroscience Education, 12(1), A42-A48.

Open Access This chapter is licensed under the terms of the Creative Commons AttributionNonCommercial-NoDerivatives 4.0 International License (http://creativecommons.org/licenses/ by-nc-nd/4.0/), which permits any noncommercial use, sharing, distribution and reproduction in any medium or format, as long as you give appropriate credit to the original author(s) and the source, provide a link to the Creative Commons license and indicate if you modified the licensed material. You do not have permission under this license to share adapted material derived from this chapter or parts of it.

The images or other third party material in this chapter are included in the chapter's Creative Commons license, unless indicated otherwise in a credit line to the material. If material is not included in the chapter's Creative Commons license and your intended use is not permitted by statutory regulation or exceeds the permitted use, you will need to obtain permission directly from the copyright holder. 